\title{
Prevalence of Myopia and Associated Risk Factors Among Primary Students in the Period of Online Study During COVID-19: A Cross-Sectional Study in Guangzhou
}

\author{
Fangyuan Chen ${ }^{1,2, \dagger}$, Xiaojuan $\mathrm{He}^{2, \dagger}$, Junjie Tang ${ }^{1,2}$, Yuanting Yang ${ }^{1,2}$, Monzer Fatfat ${ }^{1,2}$, \\ Jian Chen ${ }^{2}$, Qing Zhou ${ }^{2, *}$ \\ ${ }^{1}$ First School of Clinical Medicine, Jinan University, Guangzhou, China \\ ${ }^{2}$ Department of Ophthalmology, the First Affiliated Hospital of Jinan University, Guangzhou, China
}

Email address:

dctchenfy@163.com (Fangyuan Chen),308617459@qq.com (Xiaojuan He),jnutangjj@163.com (Junjie Tang), 1623618157@qq.com (Yuanting Yang), moonthere_f@hotmail.com (M. Fatfat),drchenj@163.com (Jian Chen), kerryzh@163.com (Qing Zhou)

${ }^{*}$ Corresponding author

$\dagger$ Fangyuan Chen and Xiaojuan He are co-first authors.

\section{To cite this article:}

Fangyuan Chen, Xiaojuan He, Junjie Tang, Yuanting Yang, Monzer Fatfat, Jian Chen, Qing Zhou. Prevalence of Myopia and Associated Risk Factors Among Primary Students in the Period of Online Study During COVID-19: A Cross-Sectional Study in Guangzhou. International Journal of Ophthalmology \& Visual Science. Vol. 5, No. 4, 2020, pp. 84-89. doi: 10.11648/j.ijovs.20200504.11

Received: October 17, 2020; Accepted: October 27, 2020; Published: November 4, 2020

\begin{abstract}
Introduction: To investigates the impact of online study during COVID-19 on myopia in Chinese primary school students and to further analyze the influencing factors of myopia, so as to provide theoretical basis for prevention and control of myopia. Objectives: Cross-sectional study. A total of 905 primary school students from grade 1-6 in Guangzhou city were included in the study. Data were collected from uncorrected visual acuity (UCVA), non-cycloplegic photo refraction and an online questionnaire. Myopia was defined as visual acuity $<1.0$ or spherical equivalent (SE) of $\leq-0.50$ diopters (D) in either eye. Chi-square and binary logistic regression analysis were applied to investigate the prevalence of myopia for different groups and potential risk factors on myopia. Results: A total of 905 students were evaluated. The mean (standard deviation) age was $9.7( \pm 1.82)$ years. 699 students were identified to have myopic refractive error making the prevalence of $77.2 \%$ ([95\% confidence interval (CI): $75.5 \%-80.0 \%$ ]; myopia prevalence significantly increased with grade level, from $61.9 \%$ (95\% CI: 54.0\%-69.8\%) at grade 1 to $84.0 \%(95 \% \mathrm{CI}$ : $80.5 \%-90.1 \%)$ at grade 6 . Low myopia $(-3.0 \mathrm{D} \leq \mathrm{SE} \leq-0.50 \mathrm{D})$ was account for $83.5 \%(95 \% \mathrm{CI}$ : $80.3 \%-86.3 \%)$ among the myopia group, with the mean (standard deviation) SE (-1.32 \pm 0.66$)$ D. Compared with children without myopia, the dry symptoms were more serious in myopic students $\left(81.8 \%\right.$ vs. $\left.25.2 \%, \chi^{2}=5.811, \mathrm{P}=0.016\right)$. Moreover, myopia prevalence significantly differences among different exposure natural light level $\left(\chi^{2}=8.382, \mathrm{P}=0.039\right)$. Binary logistic regression analysis showed children who spent more time on online-study or had higher grade level had a higher risk of myopia (OR: 1.147, 95\% CI: 1.044-1.259; OR: 1.147, 95\% CI: 1.044-1.259). Conclusions: The prevalence of myopia in primary school students from grade 1 to grade 6 in Guangzhou city is higher than previous study conducted in Guangzhou after the online-study during COVID-19. Myopia was significantly positively associated with higher grade, spending a less time outdoors; notably, playing with electronics had the greatest influence on the risk of myopia.
\end{abstract}

Keywords: Myopia, Primary School Student, Online Study, COVID-19, Cross-Sectional Study

\section{Introduction}

In the past few decades, myopia is a recognized public health concern worldwide. It is progressive and a significant major cause of visual impairment, including glaucoma, diseases of the ocular fundus, and eve blindness [1]. The prevalence of myopia is currently $28.3 \%$ worldwide, and it is predicted that by $2050,50 \%$ of the world's population will be affected by myopia [2], of which $10 \%$ will develop high 
myopia [3]. Notably, myopia is heavily affecting on school-aged, especially in East Asia, where the prevalence of myopia is now very high, with around $80 \%$ of students completing middle school [4].

Overall, although myopia imposes such a great health burden, the exact pathogenic mechanism of myopia is still unclear. The theory about both environmental and genetic factors contribute to the development of myopia is widely acknowledged recently $[5,6]$, even suggested that environmental risk factors can change the progression of myopia more rapidly than genetic factors [7]. Many environmental risk factors for schoolchildren, such as family history [8], ethnicity [9], living environment [10], near vision tasks [8], outdoor activity [8], obesity [10], socioeconomic status [10] and poor sleep quality [11], were demonstrated in previous studies.

Since the outbreak of COVID-19, Chinese primary school students have adopted online teaching and were advocated to use electric devices for school works. These changes may have substantial impact on the prevalence of myopia. Therefore, we conducted this cross-sectional study to (a) investigate the prevalence of myopia during COVID-19 on myopia and (b) to explore the relationships between refractive error data and demographic and lifestyle variables in school children grade $1-6$.

\section{Methods}

\subsection{Study Design and Population}

A total of 905 school students, ages ranging from 6-13 years old, were recruited in this cross-sectional survey, which investigated the prevalence of myopia and its associated risk factors in children attending primary schools from grades 1 to 6 in Tianhe district, Guangzhou city, China. We informed the school principals and class teachers of the related content and procedures of the study. All children were invited to participate in the study, except those with eye diseases or underwent orthokeratology lenses treatment in the past two weeks. Written informed consent forms were obtained from the children's parents before the eye examinations and questionnaires were administered. Institutional Review Board/Ethics Committee approval was obtained from the Institutional Review Board of the First Affiliated Hospital of Jinan University. All study procedures adhered to the principles outlined in the Declaration of Helsinki for research involving human subjects.

\subsection{Visual Acuity Test}

All the ocular tests were performed by an optometrist under room light during the daytime. The subjects are asked to remove the spectacle glasses for the uncorrected visual acuity test. Binocular uncorrected visual acuity (UCVA) were measured with logarithmic tumbling E chart and the child was asked to use fingers to point the direction of the opening of the "E" letter from a distance of $5 \mathrm{~m}$ and recorded as the smallest size that the child can identify in all four directions, starting with the right eye (OD) while the left eye (OS) was covered lightly with the palm of hand. Before the visual acuity test of OS, a 5-10 s of break time break was given to each child for the OS to recover from the previous occlusion. Refractometry was performed in all children in a noncycloplegic state by auto-refractometry (Topcon, Tokyo, Japan). The spherical equivalent (SE) was calculated as the spherical refractive error $+1 / 2$ of the cylindrical refractive error.

\subsection{Questionnaire Data}

For the integrity of data, questionnaires were completed by the parents and students online. The questionnaire items included:

-Demographic characteristics

Age, sex, grade level, height and weight

-Genetic factor

Parental myopia status and their education level

-Multiple online-study environmental factors

Online study duration, daily screen time, usage habits of electronic such as type of electronic equipment (smartphone/ tablet PC or TV or computer), brightness of equipment and font size of screen, whether studying in a dim environment, studying while lying down, and average reading distance to electronic.

-Daily activities and dietary habits

The number of times the child performed eye exercises daily, class recess location, daily sleep duration, the number of hours per day spent on outdoors/indoors activities and sunlight exposure. Also, the dietary habits such as frequency of eating desserts, sugary drinks, milk, vegetables and fruits, and nuts were attended.

-Others

Symptoms of dry eye and pressure

\subsection{Definition of Myopia}

In this study, myopia was defined as UCVA $<1.0$ or refractive error (spherical equivalent) at least -0.50 diopters (D) in either eye. The myopia severity was further categorized into three groups: low myopia $(\leq-0.50 \mathrm{D}$ to $\geq-3.0 \mathrm{D})$, moderate myopia ( $<-3.00 \mathrm{D}$ to $\geq-6.00)$, and high myopia $(<$ $-6.00 \mathrm{D})$, base on their non-cycloplegic auto-refraction.

\subsection{Data Analysis and Statistics}

The prevalence rates and $95 \%$ confidence intervals (CIs) of myopia were reported. Chi-square test was used to compare the distribution of myopia related conditions among students in different grades; One-way ANOVA test was used to compare the distribution of myopia diopters across different groups. All statistically significant factors in the univariate analyses were further analyzed using logistic regression models to determine the risk factors for myopia in children based on the odds ratio (OR) and 95\% CIs. Values are presented as number $(\%)$ and the mean \pm SD. Statistical analysis was performed in SPSS software (version 12; Chicago) and two-sided $p<0.05$ was considered to be statistically significant. 


\section{Results}

\subsection{Study Population}

As shown in Table 1, a total of 905 students (505 boys) were involved in this analysis, with a mean age of 9.7 years (ranged from 6 to 13 years). The mean refractive error was $-1.39( \pm 1.35) \mathrm{D}$, and the overall prevalence of myopia was $77.2 \%$ (95\% CI, 74.5\%-80.0\%] in this study. In addition, the prevalence of myopia in girls was $79.0 \%(316 / 400,95 \% \mathrm{CI}$ $75.0 \%$ to $83.0 \%)$ and in boys was $75.8 \%(383 / 505,95 \% \mathrm{CI}$ $72.1 \%$ to $79.6 \%)$, with no statistically significant $\left(\chi^{2}=1.66, \mathrm{P}\right.$ $=0.260)$. Only $19.2 \%(133 / 699)$ of students with myopia wore eyeglasses when they attended the survey.

\subsection{Descriptive Data and the Prevalence of Myopia}

The mean (standard deviation) SE of the right eye was -1.79 \pm 1.27 , and that of the left eye was $-1.54 \pm 1.43$ in myopia group. Among myopia group, refractive error varied with grade level in right eyes: mean SE was $-1.18 \mathrm{D}$ in grade 1. Mean SEs were -1.50 D, -1.39 D, -1.86 D, -1.79 D, and -2.55 $\mathrm{D}$ from grades 2 to 6 , respectively. In the left eyes, mean SEs were --0.96 D, -1.42 D, -1.21 D, -1.49 D, -1.54 D, and -2.24 D from grades 1 to 6 , respectively.

Regarding to grades, the prevalence of myopia was increased significantly, was $61.9 \%(91 / 147,95 \%$ CI $54.0 \%$ to $69.8 \%), 80.7 \%(88 / 109,95 \%$ CI $73.2 \%$ to $88.3 \%), 79.2 \%$ (122/154, 95\% CI $72.7 \%$ to $85.7 \%), 73.3 \%(99 / 135,95 \%$ CI $65.8 \%$ to $80.9 \%), 82.4 \%(140 / 170,95 \%$ CI $76.6 \%$ to $88.1 \%)$, $84.0 \%(180 / 190,95 \%$ CI $80.5 \%$ to $90.1 \%)$ respectively. (Table 1)

Showed in table 2, low myopia $(-3.0 \mathrm{D} \leq \mathrm{SE} \leq-0.50 \mathrm{D})$ was account for $83.5 \%$ (95\% CI: $80.3 \%-86.3 \%$ ) among the myopia group. The prevalence of low myopia was declined accompany with the increased of prevalence of high myopia among grade level.

Table 1. Demographic factors associated with myopia in children

\begin{tabular}{lllll}
\hline Variables & Total, n (\%) & $\begin{array}{l}\text { No myopia, n } \\
(\%)\end{array}$ & $\begin{array}{l}\text { Myopia, n } \\
(\%)\end{array}$ & P value \\
\hline $\begin{array}{l}\text { Total } \\
\text { Gender }\end{array}$ & $905(100 \%)$ & $206(22.8 \%)$ & $699(77.2 \%)$ & \\
Male & $505(100 \%)$ & $122(24.2 \%)$ & $383(75.8 \%)$ & 0.260 \\
female & $400(100 \%)$ & $84(21.0 \%)$ & $316(79.0 \%)$ & \\
Grade & & & & 0.000 \\
Grade 1 & $147(100 \%)$ & $56(38.1 \%)$ & $91(61.9 \%)$ & \\
Grade 2 & $109(100 \%)$ & $21(19.30 \%)$ & $88(80.70 \%)$ & \\
Grade 3 & $154(100 \%)$ & $32(20.80 \%)$ & $122(79.20 \%)$ & \\
Grade 4 & $135(100 \%)$ & $36(26.70 \%)$ & $99(73.30 \%)$ & \\
Grade 5 & $170(100 \%)$ & $30(17.60 \%)$ & $140(82.40 \%)$ & \\
Grade 6 & $190(100 \%)$ & $31(16.30 \%)$ & $180(84.0 \%)$ & \\
\hline
\end{tabular}

Table 2. Prevalence rate and categories of myopia.

\begin{tabular}{lllll}
\hline \multirow{2}{*}{ Variables } & Myopia (\%, 95 & \multicolumn{3}{l}{ Myopia categories n (\%) } \\
\cline { 3 - 5 } & Cl) & Low & Moderate & High \\
\hline Total & $77.2(74.5-80.0)$ & $584(83.5)$ & $105,(15.0)$ & $10(1.4)$ \\
Gender & & & & \\
Male & $75.8(72.1-79.6)$ & $320(83.6)$ & $58(15.1)$ & $5(1.3)$ \\
female & $79.0(75,0-83.0)$ & $264(83.5)$ & $47(14.9)$ & $5(1.6)$ \\
Grade & & & & \\
\hline
\end{tabular}

\begin{tabular}{lllll}
\hline \multirow{2}{*}{ Variables } & Myopia (\%, 95 & \multicolumn{3}{l}{ Myopia categories n (\%) } \\
\cline { 3 - 5 } & CI) & Low & Moderate & High \\
\hline Grade 1 & $61.9(54.0-69.8)$ & $89(97.8)$ & $2(2.2)$ & $0(0)$ \\
Grade 2 & $80.7(73.2-88.3)$ & $79(89.8)$ & $9(10.2)$ & $0(0)$ \\
Grade 3 & $79.2(72.7-85.7)$ & $115(94.3)$ & $7(5.7)$ & $0(0)$ \\
Grade 4 & $73.3(65.8-80.9)$ & $80(80.8)$ & $17(17,1)$ & $2(2.1)$ \\
Grade 5 & $82.4(76.6-81.1)$ & $118(84.3)$ & $22(15.7)$ & $0(0)$ \\
Grade 6 & $84.0(80.5-90.1)$ & $103(64.8)$ & $48(30.2)$ & $8(5.0)$ \\
\hline
\end{tabular}

Abbreviation: CI confidence interval.

\subsection{Risk Factors Identified by Logistic Regression Analysis}

A univariate analysis was employed to test the association between relevant variables with myopia in table 3 . The prevalence of myopia was significantly associated with increasing grade $(\mathrm{P}=0.000)$, more time online study $(\mathrm{P}=$ $0.018)$, espousing less natural light $(\mathrm{P}=0.039)$, spending less time outdoors $(\mathrm{P}=0.002)$ as well as shorter sleeping duration $(\mathrm{P}=0.042)$. The prevalence of myopia was not associated with sex, BMI, paternal myopia, maternal myopia, performing more eye exercises, the kind of electric, reading while lying down, reading while in a dark environment, or reading distance and dietary habits, such as the frequency of eating desserts, sugary drinks, milk, vegetables and fruits. The myopic children use mobile phone/iPad or computer or TV with rate of $75.6 \%, 80.0 \%$ and $79.1 \%$ respectively, compared to no myopia groups with rate of $24.4 \%, 20.0 \%$ and $20.9 \%$ respectively. In period of online study, the dry symptom was more serious in students with myopia than those without myopia $(\mathrm{P}=0.016)$.

Table 3. Associations between potential factors and myopia in univariate analysis.

\begin{tabular}{|c|c|c|c|c|}
\hline Variables & No myopia $(\mathbf{n}, \%)$ & Myopia (n,\%) & $\mathbf{X}^{2}$ & $\mathbf{P}$ \\
\hline \multicolumn{5}{|l|}{ Gender } \\
\hline female & $122(24.2 \%)$ & $383(75.8 \%)$ & 1.266 & 0.260 \\
\hline male & $84(21.0 \%)$ & $316(79.0 \%)$ & & \\
\hline \multicolumn{5}{|c|}{ Parental myopia } \\
\hline none & $48(24.4 \%)$ & $149(75.6 \%)$ & 1.319 & 0.517 \\
\hline one of parent & $86(23.8 \%)$ & $275(76.2 \%)$ & & \\
\hline both & $72(20.7 \%)$ & $275(79.3 \%)$ & & \\
\hline \multicolumn{5}{|c|}{ Electric device for educational } \\
\hline TV & $24(20.9 \%)$ & $91(79.1 \%)$ & 2.078 & 0.354 \\
\hline computer & $48(20.0 \%)$ & $192(80.0 \%)$ & & \\
\hline $\begin{array}{l}\text { Smart phone/ } \\
\text { IPad }\end{array}$ & $134(24.4 \%)$ & $416(75.6 \%)$ & & \\
\hline \multicolumn{5}{|c|}{ Average Duration of online study daily } \\
\hline $1-3 \mathrm{~h}$ & $154(25.8 \%)$ & $443(74.2 \%)$ & $10.123 *$ & 0.018 \\
\hline $3-5 \mathrm{~h}$ & $45(18.0 \%)$ & $205(82.0 \%)$ & & \\
\hline $5-7 \mathrm{~h}$ & $6(12.0 \%)$ & $44(88.0 \%)$ & & \\
\hline$>7 \mathrm{~h}$ & $1(12.5 \%)$ & $7(87.5)$ & & \\
\hline \multicolumn{5}{|c|}{ Average duration of online study } \\
\hline$<1 \mathrm{M}$ & $50(24.3 \%)$ & $156(75.7 \%)$ & 1.850 & 0.604 \\
\hline $1-2 \mathrm{M}$ & $86(24.2 \%)$ & $270(75.8 \%)$ & & \\
\hline $2-3 \mathrm{M}$ & $41(19.8 \%)$ & $166(80.2 \%)$ & & \\
\hline$>4 \mathrm{M}$ & $29(213 \%)$ & $107(78.7 \%)$ & & \\
\hline \multicolumn{5}{|c|}{ Reading in dim environment } \\
\hline no & $187(22.4 \%)$ & $649(77.6 \%)$ & 0.968 & 0.325 \\
\hline yes & $19(27.5 \%)$ & $50(72.5 \%)$ & & \\
\hline \multicolumn{5}{|c|}{ Keep a distance from electric device } \\
\hline no & $61(20.7 \%)$ & $233(79.3 \%)$ & 1.005 & 0.316 \\
\hline yes & $145(23.7 \%)$ & $466(76.3 \%)$ & & \\
\hline \multicolumn{5}{|c|}{ Keep a distance from writing } \\
\hline
\end{tabular}




\begin{tabular}{|c|c|c|c|c|}
\hline Variables & No myopia $(\mathbf{n}, \%)$ & Myopia (n,\%) & $\mathrm{X}^{2}$ & $\mathbf{P}$ \\
\hline no & $70(21.1 \%)$ & $262(78.9 \%)$ & 0.840 & 0.359 \\
\hline yes & $136(23.7 \%)$ & $437(76.3 \%)$ & & \\
\hline \multicolumn{5}{|c|}{ Reading while lying down } \\
\hline none & $94(23.9 \%)$ & $299(76.1 \%)$ & 0.578 & 0.902 \\
\hline occasionally & $89(22.0 \%)$ & $315(78.0 \%)$ & & \\
\hline usually & $19(21.6 \%)$ & $69(78.4 \%)$ & & \\
\hline always & $4(20.0 \%)$ & $16(80.0 \%)$ & & \\
\hline \multicolumn{5}{|c|}{ Eye recession during reading } \\
\hline no & $85(22.7 \%)$ & $289(77.3 \%)$ & 0.000 & 0.983 \\
\hline yes & $121(22.8 \%)$ & $410(77.2 \%)$ & & \\
\hline \multicolumn{5}{|c|}{ Do eye exercises } \\
\hline none & $107(23.1 \%)$ & $356(76.9 \%)$ & 0.328 & 0.955 \\
\hline occasionally & $30(22.9 \%)$ & $101(77.1 \%)$ & & \\
\hline usually & $24(20.7 \%)$ & $92(79.3 \%)$ & & \\
\hline always & $45(23.1 \%)$ & $150(76.9 \%)$ & & \\
\hline \multicolumn{5}{|c|}{ Dry eye symptom } \\
\hline no & $149(25.2 \%)$ & $442(74.8 \%)$ & 5.811 & 0.016 \\
\hline yes & $57(18.2 \%)$ & $257(81.8 \%)$ & & \\
\hline \multicolumn{5}{|c|}{ Daily hours of outdoor activity } \\
\hline none & $67(19.8 \%)$ & $271(80.2 \%)$ & $16.588^{*}$ & 0.002 \\
\hline$<1 \mathrm{~h}$ & $29(24.8 \%)$ & $88(75.2 \%)$ & & \\
\hline $1-3 \mathrm{~h}$ & $54(21.6 \%)$ & $196(78.4 \%)$ & & \\
\hline $3-5 \mathrm{~h}$ & $20(18.7 \%)$ & $87(81.3 \%)$ & & \\
\hline$>5 \mathrm{~h}$ & $36(38.7 \%)$ & $57(61.3 \%)$ & & \\
\hline \multicolumn{5}{|c|}{ Exposure to natural light } \\
\hline none & $6(16.7 \%)$ & $30(83.3 \%)$ & $8.382 *$ & 0.039 \\
\hline occasionally & $58(19.5 \%)$ & $240(80.5 \%)$ & & \\
\hline usually & $71(21.7 \%)$ & $256(78.3 \%)$ & & \\
\hline always & $71(21.7 \%)$ & $173(70.9 \%)$ & & \\
\hline \multicolumn{5}{|l|}{ Habit of sugar } \\
\hline none & $10(16.4 \%)$ & $51(83.6 \%)$ & 2.015 & 0.569 \\
\hline occasionally & $135(22.8 \%)$ & $458(77.2 \%)$ & & \\
\hline usually & $54(25.7 \%)$ & $156(74.3 \%)$ & & \\
\hline always & $7(17.1 \%)$ & $34(82.9 \%)$ & & \\
\hline \multicolumn{5}{|l|}{ Habit of drink } \\
\hline none & $74(20.8 \%)$ & $281(79.2 \%)$ & 3.240 & 0.356 \\
\hline occasionally & $116(23.5 \%)$ & $377(76.5 \%)$ & & \\
\hline usually & $16(29.6 \%)$ & $38(70.4 \%)$ & & \\
\hline always & $0(0 \%)$ & $3(100 \%)$ & & \\
\hline \multicolumn{5}{|l|}{ Habit of nut } \\
\hline occasionally & $131(21.9 \%)$ & $466(78.1 \%)$ & & \\
\hline usually & $38(24.2 \%)$ & $119(75.8 \%)$ & & \\
\hline always & $4(21.1 \%)$ & $15(78.9 \%)$ & & \\
\hline \multicolumn{5}{|l|}{ Habit of milk } \\
\hline none & $5(25.0 \%)$ & $25(75.0 \%)$ & 0.940 & 0.816 \\
\hline occasionally & $26(19.8 \%)$ & $105(80.2 \%)$ & & \\
\hline usually & $81(23.9 \%)$ & $258(76.1 \%)$ & & \\
\hline always & $206(22.8 \%)$ & $699(77.3 \%)$ & & \\
\hline \multicolumn{5}{|c|}{ Habit of vegetable } \\
\hline none & $0(0 \%)$ & $4(100 \%)$ & 3.320 & 0.345 \\
\hline occasionally & $17(18.7 \%)$ & $74(81.3 \%)$ & & \\
\hline usually & $71(21.5 \%)$ & $260(78.5 \%)$ & & \\
\hline always & $118(24.6 \%)$ & $361(75.4 \%)$ & & \\
\hline \multicolumn{5}{|c|}{ Sleep time per day } \\
\hline$<6 \mathrm{~h}$ & $0(0 \%)$ & $0(0 \%)$ & $8.198 *$ & 0.042 \\
\hline $6-8 \mathrm{~h}$ & $25(18.1 \%)$ & $113(81.9 \%)$ & & \\
\hline $8-10 \mathrm{~h}$ & $185(22.9 \%)$ & $557(77.1 \%)$ & & \\
\hline$>10 \mathrm{~h}$ & $16(38.1 \%)$ & $26(61.9 \%)$ & & \\
\hline \multicolumn{5}{|l|}{ BMI } \\
\hline$<24$ & $197(23.3 \%)$ & $648(76.7 \%)$ & 2.218 & 0.330 \\
\hline $24 \leq$ & $4(14.3 \%)$ & $24(85.7 \%)$ & & \\
\hline$>28$ & $5(15.6 \%)$ & $27(84.4 \%)$ & & \\
\hline
\end{tabular}

$\chi^{2}$ tests were used to examine the differences between myopia and no myopia group.
In binary logistic regression the prevalence of myopia as the dependent variable, which were associated with myopia prevalence in the univariate analysis, as independent variables. The results of binary logistic regression analysis demonstrated that as children spending more time on online study, their risk of myopia increased (OR: 1.390, 95\% CI: 1.043-1.851). There was strongly significant association between the grade level and the prevalence of myopia. It was shown that the prevalence of myopia in students increased along with the growth of grade level (OR: 1.147, 95\% CI: $1.044-1.259$ ). (Table 4)

Table 4. Predictors of myopia based on two-level binary logistic regression analysis.

\begin{tabular}{lllllllll}
\hline & B & SE & Wald & Sig & $\begin{array}{l}\text { Exp } \\
\text { (B) }\end{array}$ & \multicolumn{2}{c}{$\begin{array}{l}\text { 95.0\% CI. for } \\
\text { EXP(B) }\end{array}$} \\
\hline $\begin{array}{l}\text { Average duration of } \\
\text { online study daily }\end{array}$ & 0.329 & 0.146 & 5.051 & 0.025 & 1.39 & 1.043 & 1.851 \\
$\begin{array}{l}\text { Daily hours of } \\
\text { outdoor activity }\end{array}$ & -0.079 & 0.062 & 1.622 & 0.203 & 0.924 & 0.819 & 1.043 \\
$\begin{array}{l}\text { Exposure to natural } \\
\text { light }\end{array}$ & -0.176 & 0.1 & 3.083 & 0.079 & 0.839 & 0.69 & 1.021 \\
$\begin{array}{l}\text { Sleep time per day } \\
\text { Dry eye symptom }\end{array}$ & -0.285 & 0.195 & 2.145 & 0.143 & 0.752 & 0.513 & 1.101 \\
Grade level & 0.297 & 0.178 & 2.778 & 0.096 & 1.346 & 0.949 & 1.91 \\
\hline
\end{tabular}

\section{Discussion}

To the best of our knowledge, the present study is the first investigation of the prevalence of myopia and its associated risk factors among primary school children in Guangzhou after online-study in the period of COVID-19. The overall prevalence of myopia among primary school children in this study was $77.1 \%$, which the result was higher than that in some areas of China, such as reports indicated a prevalence of $33.9 \%$ in ages $9-13$ years students in Chongqing [12], 53.4\% in children aged 7-18 years in Beijing [13], and 37.7\% in grade 1-6 in Hong Kong [14]. The result was higher than figures reported from previous reports in Guangzhou, a prevalence of $47.4 \%$ among grade $1-9$ in 2016 [15]. These data suggest that myopia is a serious public health problem in Guangzhou, along with other areas of China. In addition, a prior study showed that the prevalence of aged 7-11 years children myopia in Korea was 48.2\% [16], and in the Colombia demonstrated that the prevalence of myopia in aged 8-17 years children was $11.20 \%$ [17] and in aged $13-18$ years South Africa children was only 7.0\% [18]; the prevalence of myopia in these studies were lower than that described in our study, based on non-cycloplegic measurements. Although the prevalence of myopia is high, the low myopia is account for $83.5 \%(584 / 699)$, and high myopia is account for $1.4 \%$ $(10 / 699)$.

Some surveys pointed out that the earlier the onset of myopia, the faster the progression and the higher the chance that pathologic myopia would develop [19]. We may foresee a high chance of child, has already high myopia in primary, developing sight threatening complications later in life. Thus, early intervention of myopia control in primary school 
children should be promoted, particularly in those with myopia onset at a younger age.

The evidence from a study showed that higher prevalence of myopia was associated with higher grade level, which was consistent with the result of a study done in Guangzhou which reported similar trend [15]. Hence, treatment would start at as young an age as possible so that the development of myopia can be arrested at its inception. Although a prior study reported that female children had a slightly higher risk of myopia than male [15], we did not find any significant association between sex and myopia in children in this study, this variation could be due to the difference in distribution of sex in this school.

The use of electronic devices is become a necessary require for school work during the COVID-19, and the children need to use the devices for a long time than usual. Our study demonstrated that the number of hours encountered using electronic screen products for teaching and assignments was a risk for myopia (OR: 1.147, $\mathrm{P}=0.004$ ). Near work was a is widely recognized a risk factor for myopia, supported by many surveys $[15,18]$. In the same study, Taiwanese children attending private classes outside the regular school system in the evening or on the weekends for $\geq 2 \mathrm{~h} / \mathrm{d}$ had increased risk of myopia occurrence [20]. On the other hand, a possible reason for such high prevalence rates for myopia in our study is the decrease in the amount of time spent outdoors. In this study, univariate analysis showed the prevalence of myopia was differ among the number of hours on outdoor activities. Although the results from logistic regression do not suggest any correlation between outdoors activities and myopia. According to the points of Rose [21] and Jone [22], the individuals spend more than 2 hours daily engaged in outdoor activities, the prevalence of myopia tends to decrease. If possible, the schools are to advocate to arrange the teaching and assignments reasonably and to prolong the hour exposure to natural light.

In this study, parental myopia had no association with the myopia degree in children, which is similar with the result from Hong Kong [14]. It is believed that myopia is caused by a combination of genetic and environmental factors. However, there are also suggestions that the hereditary factors transmitted from parents to children affect ocular dimensions, such as axial length, corneal radius, anterior chamber depth, rather than refraction [23]. Nonetheless, a higher proportion of myopia in children with parental myopia was identified in our study, suggesting that children with parental myopia were more likely to develop moderate or high myopia. We believe that emmetropization still continues in elementary school students, which is the reason why there was no association with spherical equivalent and parental myopia in elementary school students.

The more serious the symptoms of dry eye, the higher prevalence of myopia in the elementary school students, which was a current results in our study. Our data suggest the possibility that there could be an association between dry eye disease and myopia, this finding was similar to that in a study conducted in Japan [24]. It may related to the use of electronic screen for school work during the period of COVID-19. But it has remained unclear whether these the symptoms of dry is association with myopia. We would like to evaluate the association between these conditions in future studies. And whether long use of electronic devices might impose a negative impact on ocular health will require further evaluation.

Regarding as the durations of sleep, our study revealed that the children with $6-8$ hours or about $8-10 \mathrm{~h}$ of sleep had a higher prevalence of myopia compared to those who slept 10 hours or more daily, this finding was similar to that previous study conducted on Beijing [13]. Whether shorter time of sleep might underlying a negative impact on myopia is well not understood yet and future research is needed. It may be related with the inactivity of the ciliary muscle during the sleep or the effect of dim light and could prevent or alleviate the myopic progression [5]. New findings suggest that in addition to bright light exposure, rod pathways stimulated by dim light exposure could be important to human myopia development [25].

One study from Australia also pointed out that myopic may also have reduced sensitivity to low spatial frequency S-cone stimuli with consequences in their failure to emmetropize normally.

We have not found the differences between the prevalence of myopia and the lifestyle factors, such as the dietary habit about milk, sugar drink, and the distant from reading. We suspect that the children had

\section{Conclusions}

As a school based survey, the current study, conducted firstly during the period of COVID-19, revealed the high prevalence of myopia among primary school students aged 6 13 years in Guangzhou. Higher prevalence of myopia was significantly associated with higher grade level and longer hours spend electric device for school work. This study also indicates the need for a regular visual screening program for school children. School screening program can help in early detection and management of myopia and its associated complications. Future studies are needed on large population of students to determine the risk factors.

\section{Limitations}

1) This study was not completely reflective of the population in China because students in only one school in Guangzhou were evaluated. Future studies are needed on large population of school students to determine the risk factors.

2) The use of noncycloplegic refraction may have led to overestimation of myopia prevalence in our study.

3) The students wore contact lenses were excluded from the evaluation of the visual acuity, and the mean refraction and the prevalence in primary school would be underestimated.

4) The data, such as the dietary habit data, collected from questionnaire likely was information bias. 


\section{Abbreviation}

COVID-19: coronavirus disease 2019; UCVA: uncorrected visual acuity; SE: spherical equivalent; D: diopters; CI: confidence interval

\section{Competing Interests}

We declared that we had no competing interests.

\section{Authors' Contributions}

FY and XJ has contributed to conception, design of the study, data supervision, data entry clerk, data analyses, result interpretation, manuscript development and revision. JJ and YT and MF were responsible for reviewing the manuscript. JC and QZ were responsible for explanations of all the angiographic results and reviewing the manuscript. QZ was responsible for reviewing the manuscript. All authors read and approved the final manuscript.

\section{Acknowledgements}

We would like to thank all the staff in this article for providing valuable information.

\section{References}

[1] Resnikoff S, Pascolinia D, Mariott S P, et al. Global magnitude of visual impairment caused by uncorrected refractive errors in 2004. Bull World Health Organ, 1, 63-70 (2008).

[2] Wu P C, Huang H M, Yu H J, et al. Epidemiology of Myopia. Asia Pac J Ophthalmol (Phila), 6, 386-393 (2016).

[3] Holden B A, Fricke T R, Wilson D A, et al. Global Prevalence of Myopia and High Myopia and Temporal Trends from 2000 through 2050. Ophthalmology, 5, 1036-1042 (2016).

[4] Lin L L K, Shih Y F, Hsiao C K, et al. Prevalence of myopia in Taiwanese schoolchildren: 1983 to 2000. Ann Acad Med Singap, 1, 27-33 (2004).

[5] Grzybowski A, Kanclerz P, Tsubota K, et al. A review on the epidemiology of myopia in school children worldwide. Bmc Ophthalmol, 1 (2020).

[6] Morgan I G, French A N, Ashby R S, et al. The epidemics of myopia: Aetiology and prevention. Prog Retin Eye Res, 62, 134-149 (2018).

[7] Morgan I, Rose K. How genetic is school myopia? Prog Retin Eye Res, 1, 1-38 (2005).

[8] Low W, Dirani M, Gazzard G, et al. Family history, near work, outdoor activity, and myopia in Singapore Chinese preschool children. Br J Ophthalmol, 8, 1012-1016 (2010).

[9] Saw S M, Goh P P, Cheng A, et al. Ethnicity-specific prevalences of refractive errors vary in Asian children in neighbouring Malaysia and Singapore. Br J Ophthalmol, 10, 1230-1235 (2006).
[10] Tideman J W L, Polling J R, Hofman A, et al. Environmental factors explain socioeconomic prevalence differences in myopia in 6-year-old children. Br J Ophthalmol, 2, 243-247 (2018).

[11] Ayaki M, Torii H, Tsubota K, et al. Decreased sleep quality in high myopia children. Sci Rep, 6 (2016).

[12] Xie Z, Long Y, Wang J, et al. Prevalence of myopia and associated risk factors among primary students in Chongqing: multilevel modeling. Bmc Ophthalmol, 1 (2020).

[13] Gong Y, Zhang X, Tian D, et al. Parental myopia, near work, hours of sleep and myopia in Chinese children. Health, 06, 64-70 (2014)

[14] Choy B N K, You Q, Zhu M M, et al. Prevalence and associations of myopia in Hong Kong primary school students. Jpn J Ophthalmol, 4, 437-449 (2020).

[15] Guo L, Yang J, Mai J, et al. Prevalence and associated factors of myopia among primary and middle school-aged students: a school-based study in Guangzhou. Eye, 6, 796-804 (2016).

[16] Rim T H, Kim S-H, Lim K H, et al. Refractive Errors in Koreans: The Korea National Health and Nutrition Examination Survey 2008-2012. Korean J Ophthalmol, 3, 214-24 (2016)

[17] Galvis V, Tello A, Otero J, et al. Refractive errors in children and adolescents in Bucaramanga (Colombia). Arq Bras Oftalmol, 6, 359-363 (2017).

[18] Wajuihian S O, Hansraj R. Refractive Error in a Sample of Black High School Children in South Africa. Optom Vis Sci, 12, 1145-1152 (2017).

[19] Chua S Y L, Sabanayagam C, Cheung Y-B, et al. Age of onset of myopia predicts risk of high myopia in later childhood in myopic Singapore children. Ophthalmic Physiol Opt, 4, 388-394 (2016).

[20] Ku P-W, Steptoe A, Lai Y-J, et al. The Associations between Near Visual Activity and Incident Myopia in Children A Nationwide 4-Year Follow-up Study. Ophthalmology, 2, 214-220 (2019).

[21] Rose K A, Morgan I G, Ip J, et al. Outdoor activity reduces the prevalence of myopia in children. Ophthalmology, 8, 1279-1285 (2008).

[22] Jones L A, Sinnott L T, Mutti D O, et al. Parental history of myopia, sports and outdoor activities, and future myopia. Invest Ophthalmol Vis Sci, 8, 3524-3532 (2007).

[23] Goldschmidt E, Jacobsen N. Genetic and environmental effects on myopia development and progression. Eye, 2, 126-133 (2014).

[24] Yotsukura E, Torii H, Inokuchi M, et al. Current Prevalence of Myopia and Association of Myopia With Environmental Factors Among Schoolchildren in Japan. Jama Ophthalmol, 11, 1233-1239 (2019).

[25] Landis E G, Yang V, Brown D M, et al. Dim Light Exposure and Myopia in Children. Invest Ophthalmol Vis Sci, 12, 4804-4811 (2018). 